\title{
Designing pricing strategies for coordination of networked distributed energy resources
}

\author{
Bahman Gharesifard* Tamer Başar** \\ Alejandro D. Domínguez-García ${ }^{* *}$ \\ * Department of Mathematics and Statistics, Queen's University, \\ Kingston, Canada, bahman@mast.queensu.ca. \\ ** Coordinated Science Lab, University of Illinois, Urbana-Champaign, \\ USA, basar1@illinois.edu, aledan@illinois.edu.
}

\begin{abstract}
We study the problem of aggregator's mechanism design for controlling the amount of active, or reactive, power provided, or consumed, by a group of distributed energy resources (DERs). The aggregator interacts with the wholesale electricity market and through some market-clearing mechanism is incentivized to provide (or consume) a certain amount of active (or reactive) power over some period of time, for which it will be compensated. The objective is for the aggregator to design a pricing strategy for incentivizing DERs to modify their active (or reactive) power consumptions (or productions) so that they collectively provide the amount that the aggregator has agreed to provide. The aggregator and DERs' strategic decision-making process can be cast as a Stackelberg game, in which aggregator acts as the leader and the DERs are the followers. In previous work [Gharesifard et al., 2013b,a], we have introduced a framework in which each DER uses the pricing information provided by the aggregator and some estimate of the average energy that neighboring DERs can provide to compute a Nash equilibrium solution in a distributed manner. Here, we focus on the interplay between the aggregator's decision-making process and the DERs' decision-making process. In particular, we propose a simple feedback-based privacy-preserving pricing control strategy that allows the aggregator to coordinate the DERs so that they collectively provide the amount of active (or reactive) power agreed upon, provided that there is enough capacity available among the DERs. We provide a formal analysis of the stability of the resulting closed-loop system. We also discuss the shortcomings of the proposed pricing strategy, and propose some avenues of future work. We illustrate the proposed strategy via numerical simulations.
\end{abstract}

Keywords: Power systems, distributed energy resources, energy market, distributed control, game theory.

\section{INTRODUCTION}

Power distribution networks are undergoing radical transformation in structure and functionality. These transformations are enabled by the increased reliance on advanced communications and controls, as well as by the increased penetration of renewable-based electricity generation resources (e.g., solar photovoltaics (PV) installations), controllable loads (e.g., thermostatically-controlled loads (TCLs)), and storage-capable loads (e.g., plug-in electric vehicles $(\mathrm{PEVs})$ ). These generation resources and loads are commonly referred to as distributed energy resources (DERs), and, if properly controlled, they can be utilized to provide ancillary services. For example, PEVs and TCLs can be utilized to provide frequency regulation services [Guille and Gross, 2009, Callaway and Hiskens, 2012]. However, in order to enable the added functionality that DERs may provide, it is necessary to develop appropriate control mechanisms. In this paper, we address this problem and propose a framework for controlling the power provided/consumed by DERs, and perhaps also the reactive power if the objective is to regulate voltage.

^ This work was supported in part by a grant through the Information Trust Institute of the University of Illinois; and by NSF under grant ECCS-CPS-1135598.
Focusing on controllable loads, their control is currently achieved through demand response programs in which participants sign a contract with an aggregating entitythe demand response provider - so that their electrical energy consumption can be curtailed by the aggregator in response to market prices or in order to ensure reliable operation of the system, in exchange for lower electricity prices. In this work, we also consider an aggregating entity that will interact with the wholesale electricity market and, through pricing, will incentivize DERs to provide/consume active (or reactive) power in exchange for monetary benefits. As an example, a household with a PV system (with a reactive power capable power electronics grid interface) and a TCL might choose to offer these two resources to an aggregator so that the PV system is utilized to provide reactive power for voltage control, and the TCL is utilized to provide frequency regulation. In this sense, the interplay between the DERs and the aggregator can be modeled as a Stackelberg game, where the aggregator acts as the leader and the DERs are the followers.

\subsection{Literature Review}

Game-theoretic models have been used recently for studying energy markets (see, e.g., [Fan, 2012, Kiani and An- 
naswamy, 2012, Saad et al., 2012]). The game-theoretic parts of our work are related to noncooperative resource allocation problems in the literature, for example [Johari and Tsitsiklis, 2006, Maheswaran and Başar, 2006, Kelly et al., 1998]), where under appropriate concavity assumptions, the existence of Nash equilibrium in pure strategies is guaranteed using the results in [Rosen, 1965]. In the context of our work, the process of DER power consumption is related to the charging process in PEVs that recently appeared in [Tushar et al., 2012], and [Ma et al., 2012]; however, in our setting, we deal with a scenario in which the DERs are individual decision makers and compute the Nash equilibrium using the information available from their neighboring DERs (along with the price set by the aggregator). Additionally, in our setting, we include more general pricing strategies and allow for analysis of scenarios in which DERs are capable of both providing and consuming power.

\subsection{Statement of Contributions}

The contributions of this paper are as follows. First, building on the framework introduced in our recent work [Gharesifard et al., 2013b,a], we consider a twolayer decision-making structure. In the first layer-the aggregator layer - there is a set of aggregators that interact with the wholesale electricity market, and through some market-clearing mechanism, will enter a contract to provide a certain amount of active (or reactive) power over some predetermined period of time, for which they will be compensated. In the second layer - the retail market layer - each aggregator offers prices for the active (or reactive) power that DERs may provide or consume negative if provided and positive if consumed. Then, the objective is for the aggregator to incentivize the DERs via pricing to provide or consume active (or reactive) power so that they collectively provide an amount equal to that the aggregator has agreed to provide. We then focus on the retail market layer; specifically, on the interplay between an aggregator and a set of DERs.

We assume that each DER is a price-anticipating decisionmaker (i.e., it is aware of the aggregator pricing strategy), and in order to make a decision, it exchanges some information with neighboring DERs, with the objective of estimating the average amount of resource (active or reactive power) collectively available in its immediate neighborhood. Then, each DER uses this estimate, together with the aggregator's pricing strategy information, to decide on the amount of active (or reactive) power that it will sell or buy; this strategic decision-making process is cast as a game which, under appropriate assumptions is guaranteed to have a unique Nash equilibrium solution. When the collective active (or reactive) power consumed (or produced) by the DERs does not match the amount that the aggregator has been asked to provide, the aggregator will adjust its pricing strategy so as to steer the DERs to collectively provide the amount that the aggregator has contracted in the wholesale electricity market. In particular, we provide a feedback control mechanism that allows the aggregator to change its pricing strategy to meet its objective, i.e., to coordinate the DERs so that they collectively provide the amount of active (or reactive) power that the aggregator has agreed to provide. The upshot of this feedback control mechanism described above is that it preserves privacy, in the sense that it does not rely on the utility functions of the individual DERs.

\section{MATHEMATICAL PRELIMINARIES}

We start with some notational conventions. Let $\mathbb{R}, \mathbb{R}_{\geq 0}, \mathbb{Z}$, and $\mathbb{Z}_{>1}$ denote the set of real, nonnegative real, integer, and positive integer numbers, respectively. We denote by $\mathscr{B}(X)$ the set of bounded real-valued functions on a set $X \subset \mathbb{R}^{d}, d \in \mathbb{Z}_{\geq 1}$; we use $\mathscr{B}^{0}(X)$ when the functions are, additionally, continuous. We use the short-hand notation $\mathbf{1}_{d}=(1, \ldots, 1)^{T} \in \mathbb{R}^{d}$ and $0_{d}=(0, \ldots, 0)^{T} \in \mathbb{R}^{d}$. We also denote by $e_{i} \in \mathbb{R}^{d}$ the unit vector with 1 in its $i$ th coordinate and zeros elsewhere.

\subsection{Graph Theory}

Next, we introduce some basic notions from graph theory [Biggs, 1994]. A graph $\mathcal{G}=(\mathcal{V}, \mathcal{E})$ consists of a vertex set $\mathcal{V}$ and an edge set $\mathcal{E} \subseteq[\mathcal{V}]^{2}:=\{(v, w) \mid v, w \in$ $\mathcal{V}\}$. The neighbors of $v \in \overline{\mathcal{V}}$ belong to the set $\mathcal{N}_{v}:=$ $\{w \in \mathcal{V} \mid(v, w) \in \mathcal{E}\}$ and the degree of $v$ is $d_{v}:=$ $\left|\mathcal{N}_{v}\right|$. A path in $\mathcal{G}$ is a sequence of edges of the form $\left(v_{1}, v_{2}\right),\left(v_{2}, v_{3}\right), \ldots,\left(v_{k-1}, v_{k}\right)$. The graph $\mathcal{G}$ is connected if there is a path between any pair of vertices. Henceforth, when not explicitly stated, we fix an ordering on the vertex set $\mathcal{V}$ so that we may write $\mathcal{V}=\left\{v_{1}, \ldots, v_{n}\right\}$, where $n$ is the order of $\mathcal{G}$. The adjacency matrix of $\mathcal{G}$ is the $n \times n$ matrix $\mathrm{A}$ defined as $\mathrm{A}_{i, j}=1$ if $\left\{v_{i}, v_{j}\right\} \in \mathcal{E}$ and $\mathrm{A}_{i, j}=0$ otherwise. The weighted degree $v_{i}, i \in\{1, \ldots, n\}$ is $d\left(v_{i}\right)=\sum_{j=1}^{n} a_{i j}$. The weighted degree matrix $\mathrm{D}$ is the diagonal matrix defined by $(\mathrm{D})_{i i}=d(i)$, for all $i \in\{1, \ldots, n\}$. The Laplacian is $\mathrm{L}=\mathrm{D}-\mathrm{A}$. For an (undirected) graph, $\mathbf{L} \mathbf{1}_{n}=\mathbf{1}_{n}^{T} \mathrm{~L}=0$, and $\mathrm{L}=\mathrm{L}^{T}$ and is positive semidefinite. When $\mathcal{G}$ is connected, the zero eigenvalue is simple.

\subsection{Game Theory}

We recall the class of concave games in the absence of shared constraints, see [Rosen, 1965, Başar and Olsder, 1999]. A concave game (with unshared constraints) is a triplet $\mathbf{G}=\left(V, S,\left\{f_{i}\right\}_{i=1}^{n}\right)$, where

- $V$ is a group of $n \in \mathbb{Z}_{\geq 1}$ players,

- $S=S_{1} \times S_{2} \times \ldots \times \bar{S}_{n}$ is the product strategy set, where $S_{i} \subset \mathbb{R}^{d_{i}}$ with $d_{i} \in \mathbb{Z}_{\geq 1}$, is nonempty, convex and compact, and

- $f_{i}: S \rightarrow \mathbb{R}$, the payoff for player $i \in\{1, \ldots, n\}$, is a locally Lipschitz concave mapping.

A point $x^{*} \in S$ is called a Nash equilibrium of $G$ if and only if, for all $i \in V$,

$f_{i}\left(x^{*}\right)=\max _{y_{i}}\left\{f_{i}\left(x_{1}^{*}, \ldots, x_{i-1}^{*}, y_{i}, x_{i+1}^{*}, \ldots, x_{n}^{*}\right) \mid y_{i} \in S_{i}\right\}$.

In other words, when the game is at $x^{*}$, no player can improve its payoff by unilaterally deviating from this point. The existence of a (pure) Nash equilibrium for this class of games is guaranteed [Rosen, 1965]. A uniqueness result can also be obtained under the so-called diagonally strict concavity assumption, along with differentiability (see [Rosen, 1965, Theorem 4]), when one considers another suitable notion of equilibrium (the so-called normalized or variational equilibrium), see [Kulkarni and Shanbhag, 2012]. When the constraints are not shared, as it is the case in this paper, these notions of equilibria match, yielding an applicable uniqueness result. 


\section{PROBLEM STATEMENT}

We first recall some of the notions that were introduced in [Gharesifard et al., 2013b,a]. Consider a set of aggregators, denoted by $\left\{v_{1}^{\text {agg }}, \ldots, v_{N}^{\text {agg }}\right\}, N \in \mathbb{Z}_{\geq 1}$, who, through some market-clearing mechanism, are asked to provide a certain amount of resource (active or reactive power) over some predetermined period of time. We assume that each aggregator has a backup storage device that can be used to provide (or consume) the difference. Alternatively, one can assume that the aggregator contracts some insurance with a third party that will provide the difference. Each aggregator is responsible for controlling the amount of resource provided or consumed by a group of DERs via some pricing strategy. The collection of all DERs builds a new layer, which we term the retail market layer. The notions above are illustrated in Fig. 1, with the decisionmaking process of one of the aggregators as the focus of the paper; the decision-making of DERs in the retail market layer was discussed in [Gharesifard et al., 2013b,a], as we recall next.

\section{DERs' decision-making process}

We denote by $V=\left\{v_{1}, \ldots, v_{n}\right\}, n \in \mathbb{Z}_{\geq 1}$, the set of DERs interacting with the aggregator, where the available amount of resource of each $v_{i}, i \in\{1, \ldots, n\}$, at time $t \in \mathbb{R}_{>0}$ is denoted by $x_{i}(t) \in[0,1]$. Each DER is a decision maker and can freely choose to participate after receiving a request from its aggregator. The DERs can remain idle, or provide/absorb active (or reactive) power. The decision that each DER is faced with, among other things, depends on its own utility function, along with the pricing strategy designed by the aggregator. The DERs considered in [Gharesifard et al., 2013b,a], and also here, are price anticipating, in the sense that they are aware of the fact that the pricing is designed by the aggregator with respect to the average resource available. We also assume that in order for each DER to make its decision, it can collect information from neighboring DERs with which it can exchange information. Let us formalize these statements.

Without loss of generality, we assume that the DER is willing to participate in the resource reallocation process by setting the amount of resource that it is providing in the range $[0,1]$. The functions $P_{\mathrm{c}}:[0,1] \rightarrow \mathbb{R}_{>0}$ and $P_{\mathrm{p}}:[0,1] \rightarrow \mathbb{R}_{\geq 0}$ given by $P_{\mathrm{c}}(\bar{x}(t))$ and $P_{\mathrm{p}}(\bar{x}(t)), \bar{x}(t)=$ $\frac{1}{n} \sum_{i=1}^{n} x_{i}(t)$, denote, respectively, the price per unit of resource that the DERs pay when consuming and receive when providing. Additionally, it is reasonable to assume that the DERs are making their decisions on the amount of resource they will provide or absorb for a certain a priori fixed period of time that they are aware of. We also need to define a set of utility functions $U_{i}:[0,1] \rightarrow$ $\mathbb{R}_{>0}$, with values $U_{i}\left(x_{i}\right)$, for all $i \in\{1, \ldots, n\}$. Although our treatment allows for more general classes of utility functions, it is assumed that each $U_{i}$ is an increasing function of the available resource. Let us next describe the decision-making process that each DER is faced with. Similar to other scenarios of resource allocation problems (see, e.g., [Johari and Tsitsiklis, 2006]), each DER wishes to maximize a payoff function $f_{i}: X \times X_{\text {agg }} \rightarrow \mathbb{R}$, where $X=[0,1]^{n}$ and $X_{\text {agg }}=\mathscr{B}^{0}([0,1]) \times \mathscr{B}^{0}([0,1])$, given by

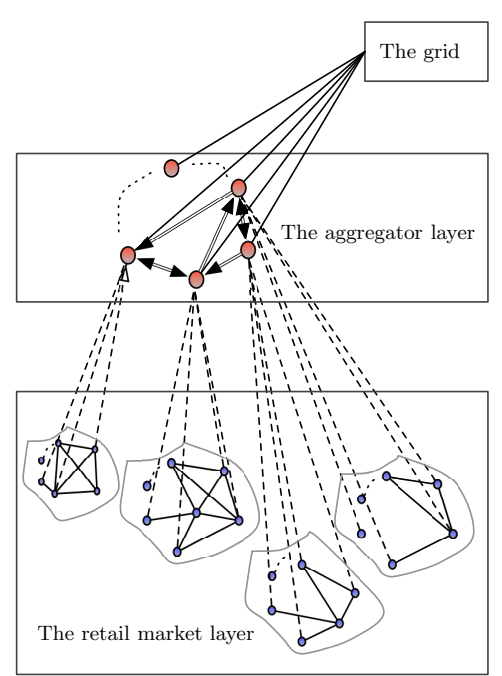

Fig. 1. Networks of DERs and their corresponding aggregators; in each network, each DER can communicate with some neighbors. DERs can also communicate with their corresponding aggregator.

$f_{i}\left(x_{i}, x_{-i}, P_{\mathrm{c}}, P_{\mathrm{p}}\right)= \begin{cases}U_{i}\left(x_{i}\right)-\left(x_{i}-x_{i}^{0}\right) P_{\mathrm{c}}(\bar{x}), & x_{i}>x_{i}^{0}, \\ U_{i}\left(x_{i}\right)-\left(x_{i}-x_{i}^{0}\right) P_{\mathrm{p}}(\bar{x}), & x_{i} \leq x_{i}^{0},\end{cases}$

where $\left(x_{i}^{0}, x_{-i}^{0}\right) \in X$ denotes the initial resource profile of all DERs.

\section{Aggregator's objective}

Let $\mathcal{X} \in \mathbb{R}$ be the amount of resource (active or reactive power) that the aggregator has contracted to provide/consume over some predetermined period of time; when $\mathcal{X} \in \mathbb{R}_{<0}$, the aggregator needs to encourage the DERs to provide active (or reactive) power. Conversely, when $\mathcal{X} \in \mathbb{R}_{>0}$, the aggregator needs to encourage the DERs to consume active (or reactive) power. The objective of the aggregator is to ensure that the DERs collectively provide $\mathcal{X} \in \mathbb{R}$ units of resource; thus it wishes to maximize the function $f_{\text {agg }}: X \times X_{\text {agg }} \rightarrow \mathbb{R}_{\leq 0}$ given by

$$
f_{\text {agg }}\left(x, P_{\mathrm{c}}, P_{\mathrm{p}}\right)=-\left|\mathcal{X}-\sum_{i=1}^{n} \alpha_{i}\left(x_{i}-x_{i}^{0}\right)\right|,
$$

where $\alpha_{i} \in \mathbb{R}_{>0}$, for all $i \in\{1, \ldots, n\}$, over the choices of $P_{\mathrm{c}}$ and $P_{\mathrm{p}}$.

Based on the description given above, the aggregator and the DERs define a game, called the retail market game,

$\mathbf{G}_{\text {DERs-AGG }}=\left(V \cup\left\{v^{\text {agg }}\right\}, \mathbb{R}^{n} \times X_{\text {agg }}, f_{1} \times \ldots \times f_{n} \times f_{\text {agg }}\right)$, where players wish to maximize their objective functions. For most part, we think of $\mathbf{G}_{\text {DERs-AGG }}$ as a two-stage Stackelberg game, where the aggregator sets the pricing strategies first and the DERs act next.

We next raise some questions, where (a-c) were previously introduced in [Gharesifard et al., 2013b,a].

(a) (Existence of equilibria): given the pricing strategies of the aggregator $P_{\mathrm{c}}, P_{\mathrm{p}} \in \mathscr{B}^{0}([0,1])$, does there exist a Nash equilibrium solution to the retail market game? If so, is the equilibrium unique? 
(b) (Distributed equilibria seeking): if the answers to both parts of (a) are positive, can the DERs use a strategy which only relies on local information available to each DER, to seek the Nash equilibrium, after the pricing strategy is fixed?

(c) (Regulating pricing strategy): if the answer to the existence part of (a) is positive, does there exist pricing strategies $P_{\mathrm{c}}, P_{\mathrm{p}} \in \mathscr{B}^{0}([0,1])$ such that

$$
x^{*} \in\left\{z \in X \mid z=\operatorname{argmax}_{x} f_{\text {agg }}\left(x, P_{\mathrm{c}}, P_{\mathrm{p}}\right)\right\}
$$

and $x^{*}$ is the retail market game Nash equilibrium?

(d) (Optimal regulating pricing strategy): What are the pricing policies that enable the aggregator to incentivize the DERs so that they collectively provide the amount agreed upon, while minimizing the cost function

$$
\begin{aligned}
f_{\text {agg }}\left(x, P_{\mathrm{c}}, P_{\mathrm{p}}\right)= & \int_{t=0}^{\infty}\left[x(t)-x^{*}\right)^{T} Q\left(x(t)-x^{*}\right) \\
& +R_{c} P_{c}^{2}(\bar{x}(t))+R_{d} P_{d}^{2}(\bar{x}(t)] d t,
\end{aligned}
$$

where $Q \in \mathbb{R}^{n \times n}$ and $R_{c}, R_{d} \in \mathbb{R}_{>0}$ ?

We have provided solutions to (a) and (b) in [Gharesifard et al., 2013b,a]; here we provide an answer to (c), and discuss possible approaches to (d).

\section{DISTRIBUTED ALGORITHMS FOR SEEKING AN EQUILIBRIUM OF THE RETAIL MARKET GAME}

We start by stating some assumptions on the payoff functions of the players.

Assumption 4.1. (Properties of the payoff functions): We assume that

(i) $U_{i}$ are concave, nondecreasing, and continuously differentiable, for all $i \in\{1, \ldots, n\}$,

(ii) $P_{\mathrm{c}}$ is convex, twice differentiable, and nondecreasing,

(iii) $P_{\mathrm{p}}$ is concave, twice differentiable, nondecreasing, and

(iv) $P_{\mathrm{c}}(\bar{x}) \geq P_{\mathrm{p}}(\bar{x})$, for all $\bar{x} \in[0,1]$.

Let us discuss some of the results obtained in [Gharesifard et al., 2013b,a] about the retail market layer, after the pricing strategy is chosen by the aggregator. First, by imposing Assumption 4.1, the retail market game is a concave game and hence has at least one pure Nash equilibrium. Moreover, when this Nash equilibrium is unique (diagonally strict concavity), it can be found using a distributed update scheme, implemented by DERs only using their local information. Note that the function $f_{i}$, although locally Lipschitz, is not necessarily differentiable. In spite of this, as we have shown in [Gharesifard et al. 2013b,a], the DERs can still follow a set-valued gradient dynamical system to arrive at a Nash equilibrium of the retail market game, when this equilibrium is unique.

Let us briefly recall this scheme, here in continuous-time. In order to make the presentation simpler, and given that a precise version of this algorithm is discussed elsewhere, we write this scheme for differentiable points of the action space. In fact, for the special case where $P_{\mathrm{c}}$ and $P_{\mathrm{p}}$ are the same (hence linear, by Assumption 4.1(ii) and (iii)), differentiability holds everywhere.

Let $\mathcal{C}=\left(X^{n} \times \mathbb{R}^{n}\right)$ and consider the dynamics $\Xi: \mathcal{C} \times$ $\left(\mathbb{R}^{n} \times \mathbb{R}^{n}\right) \rightarrow \mathcal{C} \times\left(\mathbb{R}^{n} \times \mathbb{R}^{n}\right)$ given by

$$
\begin{aligned}
\dot{\boldsymbol{x}}(t) & =\Pi_{\mathcal{C}}\left(-\mathbf{L} \boldsymbol{x}(t)-\mathbf{L} \boldsymbol{z}(t)+\boldsymbol{s}_{\boldsymbol{x}}\right), \\
\dot{\boldsymbol{z}}(t) & =\mathbf{L} \boldsymbol{x}(t),
\end{aligned}
$$

where

$$
\boldsymbol{s}_{\boldsymbol{x}}=\underbrace{\left(\nabla_{x_{1}} f_{1}\left(\boldsymbol{x}^{1}\right), 0, \ldots, 0\right.}_{\text {computed by } v_{1}}, \ldots, \underbrace{\left.0, \ldots, 0, \nabla_{x_{n}} f_{n}\left(\boldsymbol{x}^{n}\right)\right)^{T}}_{\text {computed by } v_{n}}\}\}
$$

$\mathbf{L}=\mathbf{L} \otimes \mathbf{I}_{n} \in \mathbb{R}^{n^{2} \times n^{2}}$, and $\mathbf{L}$ is the Laplacian of $\mathcal{G}_{\text {DERs }}$. Here, $\Pi_{\mathcal{C}}$ is the projection map on $\mathcal{C} \times\left(\mathbb{R}^{n} \times \mathbb{R}^{n}\right)$. We will occasionally refer to these dynamics as the concave Nashseeking dynamics. Note that (2) is clearly distributed over the network $\mathcal{G}_{\mathrm{DERs}}$ and each player only uses the information about its own payoff function. The following result was proved in [Gharesifard et al., 2013a]. Here, and also throughout the paper, we assume that the pricing strategies and the utility functions are such that diagonally strict concavity assumption holds.

Theorem 4.2. (Asymptotic convergence of (2)): Given a pricing strategy for the aggregator, when $\mathcal{G}_{\text {DERs }}$ is undirected and connected, the dynamics in (2) are asymptotically convergent. Moreover, the projection onto the first component of its trajectory converges to $\boldsymbol{x}^{*}=\mathbf{1}_{n} \otimes x^{*}$, where $x^{*} \in \mathbb{R}^{n}$ is the Nash equilibrium of $\mathbf{G}_{\text {DERs-AGG }}$.

\section{PRICING STRATEGY DESIGN FOR THE AGGREGATOR}

In this section, we discuss a simple strategy that allows the aggregator to regulate the consumption (or provision) of active (or reactive) power. It is important to note that, in general, the aggregator does not have any access to DERs' private information. As we will see, this assumption places a restriction on the class of pricing strategies that can be designed; in particular, the pricing offered might be far from "optimal" (see problem statement (d)). In this sense, the pricing strategy designed below is only implementable when there exist enough resources within the retail market so that providing the request from the grid is feasible, c.f. Remark 5.3.

Throughout this section, for simplicity and also for ensuring differentiability, we assume that the pricing functions $P_{\mathrm{c}}$ and $P_{\mathrm{p}}$ are the same, and hence linear; we denote this pricing strategy by $P$. The aggregator designs the price of consumption (or provision) $P:[0,1] \rightarrow \mathbb{R}_{\geq 0}$ as

$$
P(\bar{x}(t))=a \bar{x}(t)+u(t)
$$

where $a \in \mathbb{R}_{\geq 0}$ and $u(t) \in \mathbb{R}_{\geq 0}$ is the aggregator's control input, for all $t \in \mathbb{R}_{>0}$. For example, the aggregator may wish to use $u$ to prevent the DERs from overconsumption, when they have high utility functions. Using (1), one can write (2) as

$$
\begin{aligned}
\dot{\boldsymbol{x}}(t) & =F(\boldsymbol{x}(t), \boldsymbol{z}(t))+G u(t), \\
\dot{\boldsymbol{z}}(t) & =\mathbf{L} \boldsymbol{x}(t),
\end{aligned}
$$

where $G=\left(e_{1}, \ldots, e_{n}\right)^{T} \in \mathbb{R}^{n^{2}}$ and

$$
F(\boldsymbol{x}(t), \boldsymbol{z}(t))=\Pi_{\mathcal{C}}\left(-\mathbf{L} \boldsymbol{x}(t)-\mathbf{L} \boldsymbol{z}(t)+\boldsymbol{s}_{\boldsymbol{x}}\right)-G u(t) .
$$

Our main result is stated next.

Theorem 5.1. (Stabilizing to the aggregator's total request): Suppose that the aggregator's policy is given by (3), where $a \in \mathbb{R}_{\geq 0}$, and there exists a constant controller $u(t)=k_{\mathrm{d}}, k_{\mathrm{d}} \in \mathbb{R}_{\geq 0}$, under which the system (4) stabilizes to $\left(\boldsymbol{x}^{*}, \boldsymbol{z}^{*}\right)$, where $\boldsymbol{x}^{*}=\mathbf{1}_{n} \otimes x^{*}, x^{*} \in \mathbb{R}^{n}$, and $\mathbf{1}_{n}^{T}\left(x_{i}^{*}-x_{i}^{0}\right)=\mathcal{X}$. Then the projection onto the first and second components of any trajectory of 


$$
\begin{aligned}
\dot{\boldsymbol{x}}(t) & =F(\boldsymbol{x}(t), \boldsymbol{z}(t))+G \xi(t), \\
\dot{\boldsymbol{z}}(t) & =\mathbf{L} \boldsymbol{x}(t), \\
\dot{\xi}(t) & =-\left(\sum_{i=1}^{n}\left(x_{i}(t)-x_{i}^{0}\right)-\mathcal{X}\right),
\end{aligned}
$$

asymptotically converges to $\left(\boldsymbol{x}^{*}, \boldsymbol{z}^{*}\right)$.

Proof. For simplicity of notation, let us introduce

$$
\eta(t)=-\left(\sum_{i=1}^{n}\left(x_{i}(t)-x_{i}^{0}\right)-\mathcal{X}\right)
$$

where $\left(x_{1}, \ldots, x_{n}\right) \in \mathbb{R}^{n}$ is the resource profile of the DERs. For an initial condition $\left(\boldsymbol{x}^{0}, \boldsymbol{z}^{0}, \xi_{0}\right)$, consider the candidate Lyapunov function $V_{1}: X^{n} \times \mathbb{R}^{n} \times \mathbb{R} \rightarrow \mathbb{R}_{\geq 0}$ given by

$$
V_{1}(\boldsymbol{x}, \boldsymbol{z}, \xi)=V_{0}(\boldsymbol{x}, \boldsymbol{z})+\frac{1}{2}\left(\xi-k_{\mathrm{d}}\right)^{2},
$$

where $V_{0}$ is a quadratic control Lyapunov function for (2) given by

$$
V_{0}(\boldsymbol{x}, \boldsymbol{z})=\frac{1}{2}\left(\boldsymbol{x}-\boldsymbol{x}^{*}\right)^{T}\left(\boldsymbol{x}-\boldsymbol{x}^{*}\right)+\frac{1}{2}\left(\boldsymbol{z}-\boldsymbol{z}^{*}\right)^{T}\left(\boldsymbol{z}-\boldsymbol{z}^{*}\right),
$$

with the stabilizing feedback $P(\bar{x}(t))=a \bar{x}(t)+k_{\mathrm{d}}$. The function $V_{1}$ is clearly a smooth mapping. Let us examine its set-valued Lie derivative.

$$
\begin{aligned}
\mathcal{L}_{\Psi} V_{1}(\boldsymbol{x}, \boldsymbol{z}, \xi) & ={\frac{\partial V_{0}}{\partial \boldsymbol{x}}}^{T}(F(\boldsymbol{x}(t), \boldsymbol{z}(t))+G \xi(t))+ \\
& +{\frac{\partial V_{0}}{\partial \boldsymbol{z}}}^{T} \mathbf{L} \boldsymbol{x}(t)+\left(\xi(t)-k_{\mathrm{d}}\right) \eta(t) \\
& ={\frac{\partial V_{0}}{\partial \boldsymbol{x}}}^{T}\left(F(\boldsymbol{x}(t), \boldsymbol{z}(t))+G k_{\mathrm{d}}\right)+{\frac{\partial V_{0}}{\partial \boldsymbol{z}}}^{T} \mathbf{L} \boldsymbol{x}(t) \\
& +\left(\xi(t)-k_{\mathrm{d}}\right) \eta(t)+{\frac{\partial V_{0}}{\partial \boldsymbol{x}}}^{T} G\left(\xi(t)-k_{\mathrm{d}}\right) \\
& =\mathcal{L}_{\Phi} V_{0}(\boldsymbol{x}, \boldsymbol{z})+\left(\xi(t)-k_{\mathrm{d}}\right)\left(\eta(t)+{\frac{\partial V_{0}}{\partial \boldsymbol{x}}}^{T} G\right) .
\end{aligned}
$$

By definition of $G$, we have that $\eta(t)+{\frac{\partial V_{0}}{\partial \boldsymbol{x}}}^{T} G=0$. Given that $\mathcal{L}_{\Phi} V_{0}(\boldsymbol{x}, \boldsymbol{z}) \leq 0$, we conclude that

$$
\mathcal{L}_{\Psi} V_{1}(\boldsymbol{x}, \boldsymbol{z}, \xi) \leq 0
$$

and hence (5) is stable and has bounded trajectories. The application of the LaSalle invariance principle leads to the conclusion that, from a given initial condition, the evolution of (5) approaches a set $M$ of the form $V_{1}^{-1}(c) \cap$ $S, c \in \mathbb{R}$, where $S$ is the largest invariant set contained in

$$
\left\{(\boldsymbol{x}, \boldsymbol{z}, \xi) \in V_{1}^{-1}\left(\leq V_{1}\left(\boldsymbol{x}^{0}, \boldsymbol{z}^{0}, \xi_{0}\right)\right) \mid \mathcal{L}_{\Psi} V_{1}(\boldsymbol{x}, \boldsymbol{z}, \xi)=0\right\}
$$

Note, however, that when $\mathcal{L}_{\Psi} V_{1}(\boldsymbol{x}, \boldsymbol{z}, \xi)=0$, we have that $\mathcal{L}_{\Phi} V_{0}(\boldsymbol{x}, \boldsymbol{z})=0$, which implies that $(\boldsymbol{x}, \boldsymbol{z})=\left(\boldsymbol{x}^{*}, \boldsymbol{z}^{*}\right)$.

Remark 5.2. (Implementation of the continuoustime controller): Although we have designed the pricing inputs as a continuous-time controller, it can potentially be implemented in a discrete-time manner; this is a reasonable assumption as the change in pricing happens much slower than the Nash seeking dynamics in the retail market. A real-time scheduling can also be achieved using an event-triggered implementation [Tabuada, 2007]. Finally, it is worth mentioning that the procedure proposed is robust, a property of the saddle-point dynamical systems, and can regulate slow time-varying requests.

Remark 5.3. (Optimal regulating pricing strategy): The shortcoming of the pricing strategy provided here is that it does not take into account the revenue that

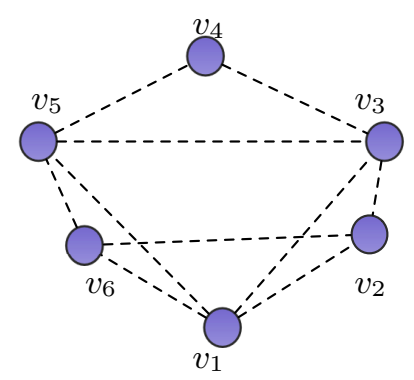

Fig. 2. The network of DERs of the simulations is shown. The adjacency matrix associated with this network has 1 in the entries corresponding to edges and zeros elsewhere.

\begin{tabular}{|c|c|c|c|c|c|c|}
\hline & DER 1 & DER 2 & DER 3 & DER 4 & DER 5 & DER 6 \\
\hline$u^{1}$ & 0.4021 & 0.7126 & 0.5502 & 0.6002 & 0.8216 & 0.7984 \\
$u^{2}$ & 0.1000 & 0.1000 & 0.1000 & 0.1000 & 0.1162 & 0.1000 \\
\hline
\end{tabular}

Table 1. The utility properties of each DER in Fig. 2.

the aggregator obtains. This is because the aggregator typically receives a fixed price through the contract in the wholesale market, but this price might be lower than the one that the aggregator needs to pay the DERs. Problem statement (d) takes this into account, by modeling the aggregators' decision-making process as the leader of a Stackelberg game. The difficulty, however, is in designing an implementable optimal solution for the aggregator which does not rely on the private information about the utility functions of the DERs. The approach developed in [Shen and Başar, 2007] provides promising avenues for addressing this.

\section{SIMULATIONS}

Consider a group of DERs connected to an aggregator; for illustration purposes, we have only selected six DERs $\left\{v_{1}, \ldots, v_{6}\right\}$. The DERs are price anticipating and can obtain information from each other via a communication network shown in Fig. 2.

Each DER's utility function $U_{i}:[0,1] \rightarrow \mathbb{R}_{\geq 0}, i \in$ $\{1, \ldots, 6\}$, is given by

$$
U_{i}(x)=u_{i}^{1} \log (1+x)+u_{i}^{2} x
$$

where $U_{i}$ is normalized so that $u_{i}^{1}, u_{i}^{2} \in(0,1]$. The values of these parameters for each DER are given in Table 1 . Note that $U_{i}$ is increasing and strictly concave, and thus satisfies Assumption 4.1(i). The pricing strategy for the aggregator is given by (3) and is thus linear. The parameter $a$ is chosen to be 0.1 here. It can be shown that with the given parameters, the diagonally strict concavity assumption holds.

Fig. 3 demonstrates the evolutions of the dynamics (5) from the initial condition $x_{0}=(0.2,0.3,0.3,0.4,0.1,0.2)^{T}$. In the first scenario, see (a) and (b), the aggregator is requested to encourage the DERs to consume resource such that $50 \%$ of their total capacity is full. The pricing input of the aggregator is shown in Fig. 4(a). In contrast, in Fig. 3(c) and (d), the aggregator encourages the DERs to provide one-third of their available resource back to the grid. Fig. 4(b) shows the pricing input of the aggregator for this case. The pricing control inputs of this example can be implemented in discrete-time, where the aggregator implements changes in its pricing scheme in a longer period of time, using its information about the average available resource within the DERs. 


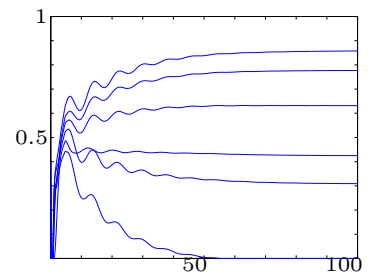

(a)

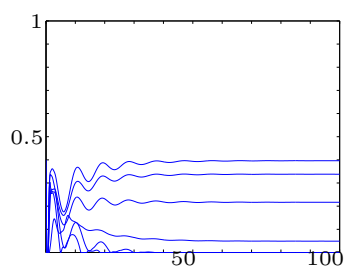

(c)

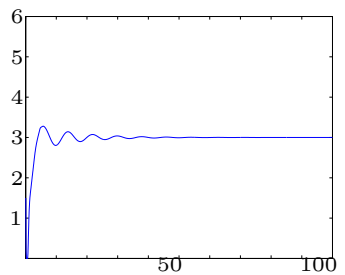

(b)

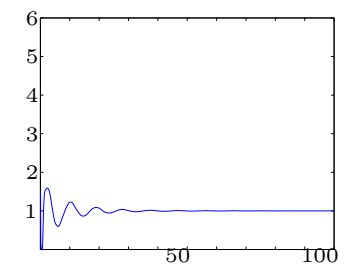

(d)
Fig. 3. (a) and (b) correspond to the scenario where the grid has requested that the aggregator encourages the DERs to consume resource such that $50 \%$ of their total capacity is used. The DERs' initial charge is $x_{0}=(0.2,0.3,0.3,0.4,0.1,0.2)^{T}$, i.e., $25 \%$ of their total capacity is used. Panel (a) shows each DERs' consumption, and Panel (b) shows their total capacity, regulated by the aggregator. In contrast, (c) and (d) correspond to the scenario where the grid has requested that the aggregator encourages the DERs to return one-third of their available resource (active or reactive power) to the grid.

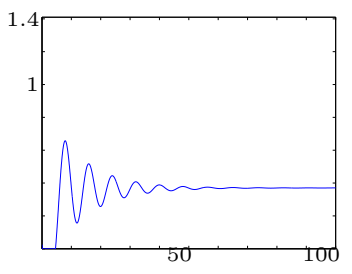

(a)

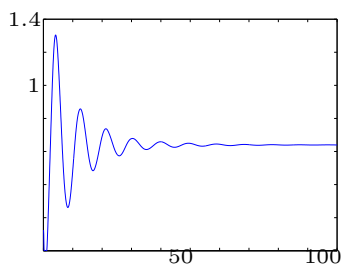

(b)
Fig. 4. (a) and (b) show the pricing control input of the aggregator, asymptotically converging to 0.3704 and 0.6396 , respectively.

\section{CONCLUSIONS AND FUTURE WORK}

We have studied the problem of incentivizing a group of DERs to provide/consume active or reactive power in exchange for monetary benefits. Building on the framework previously introduced in [Gharesifard et al., 2013b,a], the DERs considered are price anticipating and only have access to local information, but can still compute an equilibrium solution once the pricing strategy is fixed.

We have proposed a pricing strategy that allows the aggregator to regulate the consumption (or provision) of active (or reactive) power by a group of DERs. The upshot of the pricing strategy proposed is that it does not rely on the private information about the utilities of the DERs.

We have also discussed a possible shortcoming of this strategy regarding the revenue of the aggregator, and have proposed a framework for addressing it. It is an interesting avenue of research to study this problem for classes of nonlinear pricing strategies as in [Shen and Başar, 2007], where the aggregator also uses the average-dependent pricing terms for regulation.

\section{REFERENCES}

T. Başar and G. J. Olsder. Dynamic Noncooperative Game Theory. SIAM, 2nd edition, 1999.

N. Biggs. Algebraic Graph Theory. Cambridge University Press, 2 edition, 1994. ISBN 0521458978.

D. S. Callaway and I. A. Hiskens. Achieving controllability of electric loads. Proceedings of the IEEE, 3(1): 434-442, 2012.

Z. Fan. A distributed demand response algorithm and its applications to PHEV charging smart grids. IEEE Transactions on Smart Grids, 3:1280-1290, 2012.

B. Gharesifard, T. Başar, and A. D. Domínguez-García. A price-based approach to control of networked distributed energy resources. IEEE Transactions on Automatic Control, 2013a. Submitted.

B. Gharesifard, T. Başar, and A. D. Domínguez-García. Price-based distributed control for networked plug-in electric vehicles. In American Control Conference, pages 5093-5098, Washington, DC, 2013b.

C. Guille and G. Gross. A conceptual framework for the vehicle-to-grid (V2G) implementation. Energy Policy, 37(11):4379-4390, 2009.

R. Johari and J. N. Tsitsiklis. A scalable network resource allocation mechanism with bounded efficiency loss. IEEE Journal on Selected Areas in Communications, 24:992-999, 2006.

F. Kelly, A. Maulloo, and D. Tan. Rate control for communication networks: Shadow prices, proportional fairness and stability. Journal of Operations Research, 49(3):237-252, 1998.

A. Kiani and A. Annaswamy. Wholesale energy market in a smart grid: A discrete-time model and the impact of delays. Power Electronics and Power Systems, 3: 87-110, 2012.

A. A. Kulkarni and U. V. Shanbhag. On the variational equilibrium as a refinement of the generalized nash equilibrium. Automatica, 48(1):45-55, 2012.

Z. Ma, D. S. Callaway, and I. A. Hiskens. Decentralized charging control of large populations of plug-in electric vehicles. IEEE Transactions on Control Systems Technology, 2012. To appear.

R.T. Maheswaran and T. Başar. Efficient signal proportional allocation (ESPA) mechanisms: Decentralized social welfare maximization for divisible resources. $J$. of Selec. Areas in Comm., 24:1000-1009, 2006.

J. B. Rosen. Existence and uniqueness of equilibrium points for concave $n$-person games. Econometrica, 33 (3):520-534, 1965.

W. Saad, Z. Han, H. V. Poor, and T. Başar. Game theoretic methods for the smart grid. IEEE Signal Processing Magazine. Special Issue: Signal Processing Techniques for Smart Grid, 29(5):86-105, 2012.

H. Shen and T. Başar. Optimal nonlinear pricing for a monopolistic network service provider with complete and incomplete information. IEEE Journal on Selected Areas of Communications, 25(6):1216-1223, 2007.

P. Tabuada. Event-triggered real-time scheduling of stabilizing control tasks. IEEE Transactions on Automatic Control, 52(9):1680-1685, 2007.

W. Tushar, W. Saad, H. V. Poor, and D. B. Smith. Economics of electric vehicle charging: a game theoretic approach. IEEE Transactions on Smart Grids, 3(4): 1767-1778, 2012. 